\title{
Editorial
}

iMedPub Journals

http://www.imedpub.com

DOI: $10.21767 / 2471-8416.100001$

\section{Welcome to the Journal of Clinical and Experimental Orthopaedics}

\section{Chunfeng Zhao}

Department of Orthopedic Surgery, Mayo Clinic, USA

Received: September 18, 2015; Accepted: September 23, 2015; Published: September 28, 2015

Publishing timely your research in a right journal is important for your clinical or research career. Orthopedic field is a growing area in medicine, involves millions of patient, and cost huge economic burden. Your research in this regards would have a significant impact on both basic research and clinical practice. Journal of Clinical and Experimental Orthopaedics (JCEOP), a new peerreviewed and open access journal, provides rapid and reliable source of information in the mode of original articles, review articles, case reports, short communications, etc. in all areas of the field and making them freely available through online without any restrictions or any other subscriptions to researchers worldwide.

The scope of the JCEOP is to provide a scientific communication platform to allow clinicians and scientists to share their ideas,
Citation: Zhao C. Welcome to the Journal of Clinical and Experimental Orthopaedics. J Clin Exp Orthop.2015, 1:1.

present their innovations, and exchange their clinical or experimental experiences and outcomes. JCEOP focuses on the areas such as age and cancer related bone degeneration, orthopedic oncology, pediatric orthopedics, osteoporosis, foot and ankle surgery, knee replacements, hip replacement, osteopenia, joint replacement treatment of arthritic, etc. Although several important topics are mentioned, yet the journal will not be limiting the consideration for publication, other allied topics will be considered if found suitable under the wide scope of the journal, which is clearly described in the journal website.

I welcome you to join us and look forward to your submission! 